DOI 10.4467/2543733XSSB.17.007.9982

ANNA CITKOWSKA-KIMLA

Jagiellonian University

\title{
GOLO MANNS KONZEPTION DER GESCHICHTSSCHREIBUNG
}

Was dem Einzelnen die Vernunft, ist für die Menschheit die Geschichte. Arthur Schopenhauer (Golo Manns Lieblingsphilosoph)

Erst die Geschichte lehrt den Menschen, wer er ist. Wilhelm Dilthey

Traditionen sind keineswegs das Privileg konservativer Kräfte. Es kommt darauf an, auf welche Traditionen man sich bezieht und welchen Sinn die Erwähnung eines historischen Ereignisses hat.

Gustav Heinemann

\section{Golo Mann's approach to history}

\section{Summary}

The article explores the originality of Golo Mann's approach to history. The exeptional position of history in his works is analyzed based on two questions - the affinity between history and literature and the condition of historiography in a democratic system. The main thesis oft he article is showing the important role that history plays in anticipating future (also political) events.

Keywords: Golo Mann, history, politics, democracy, literature, historiography

\section{Einführung}

Das 20. Jahrhundert brachte nicht sehr viele visionäre Geschichtsbilder, ebenso selten sind Beschreibungen der Kunst der Geschichtsschreibung. Daher ist die Problematik, der sich Golo Mann in seinem Essay Geschichtsschreibung als Literatur in dem Band Menschen des Gedankens, Menschen der Macht, Geschichte ${ }^{1}$ widmet, von besonderer Bedeutung. In

\footnotetext{
${ }^{1}$ Der Titel stammt von der Übersetzerin von Golo Manns Essays ins Polnische.
} 
Polen erschien dieses Buch 1979, übersetzt und herausgegeben von Elżbieta Paczkowska-Łagowska ${ }^{2}$, einer bedeutenden Kennerin der deutschen Philosophie.

Das Hauptziel ihrer Überlegungen ist die Rekonstruktion der Ansichten des Autors des Wallenstein über die Methode der Geschichtsschreibung, was wiederum einem zweiten Zweck dient: der Darstellung der Außergewöhnlichkeit von Golo Manns Denken über die Aufzeichnung von Geschichte.

Professor Mann verknüpfte historisches und politisches Wissen. In einem seiner Interviews bekannte er sich zu seiner politischen Leidenschaft: „Von jung auf, als ich um die 16 Jahre alt war, verfolgte ich die Politik mit starkem, leidenschaftlichem und zuweilen schmerzlichem Interesse. Häufig sah ich auch den Zusammenhang zwischen meinen historiografischen Bemühungen und den Angelegenheiten der Politik. Man kann sagen, dass ich meine Teilhabe an der Gegenwart in einigen Texten auf die Vergangenheit übertrug"’3.

Bevor wir aber zu Golo Manns Gedanken kommen, lohnt es sich, zunächst diesen Historiker kurz vorzustellen, der sicherlich nicht so bekannt ist wie sein Vater Thomas $\mathrm{Mann}^{4}$, auch wenn seine intensive Arbeit mit zahlreichen Preisen bedacht wurde, Der Autor der Abhandlung Vom Geist Amerikas wurde unter anderem mit den nach Theodor Fontane, Schiller, Büchner, Gottfried Keller, Ernst-Robert Curtius oder Goethe bezeichneten Priesen ausgezeichnet.

\section{Golo Mann im „Dossier”}

Wenn diese Ausführungen Thomas Mann gelten würden, würde sich eine Vorstellung erübrigen. Da es aber um dessen Sohn Golo geht, soll die Figur dieses genialen Porträtisten historischer Persönlichkeiten eingangs kurz skizziert werden ${ }^{5}$.

Angelus Gottfried Thomas Mann war der zweite Sohn von Thomas Mann ${ }^{6}$, „Die Bande dieser einzigartigen, einander eher tolerierenden als unterstützenden Familie, vor der es jedoch keine Flucht gab, beschränkte und erstickte ihn über viele Jahre hinweg"7. Geboren wurde er 1909 in München. Er studierte Philosophie und Geschichte in München, Berlin, Paris und Heidelberg. 1932 verteidigte er bei Karl Jaspers seine Dissertation über die Philosophie Hegels. Die Geschehnisse des Jahres 1933 zwangen ihn zur Emigration. Er ging

\footnotetext{
${ }^{2}$ Grundlage der Übersetzung waren folgende Werke Golo Manns: Zeiten und Figuren. Schriften aus vier Jahrzehnten; Wissen und Trauer. Historische Portraits und Skizzen; Zwölf Versuche; Geschichtsschreibung als Literatur.

${ }^{3}$ G. Mann, G. Gaus, Nienawidzę wszelkich skrajności (Ich hasse jegliche Extremismen), übers. K. Zajas, „Przegląd Polityczny” Nr. 99/2010, S. 99.

${ }^{4}$ Welch wichtige Rolle Thomas Mann für die ganze Familie einnahm, zeigen Golos Worte über die Kriegszeit, als seine Mutter Katia Mann geradezu als Heldin schien, die beschäftigt war, ,ihren nervösen, hart arbeitenden Gatten zu beschützen, ihn so gut es ging zu ernähren und dabei dafür zu sorgen, dass auch die Übrigen nicht verloren gehen: die vier Kinder und die drei Mädchen". I. und W. Jens, Pani Tomaszowa Mann (Frau Thomas Mann), übers. E. Kowynia, Warszawa 2006, S. 81.

${ }^{5}$ Vgl. T. Lahme, Golo Mann: Biographie, Frankfurt a.M. 2009.

${ }^{6}$ Die Persönlichkeit des Vaters schlug sich auf alle Kinder nieder. Golo Mann fühlte sich von ihm abgelehnt. Mit Sicherheit ignorierte und erniedrigte der Vater ihn. Daher nimmt es nicht wunder, dass Golos Schaffenskraft mit ganzer Kraft erst nach dem Tode des Vaters 1955 zum Vorschein kam.

7 J. Fest, Szczęście jako zastuga (Glück als Verdienst), übers. A. und A. Kluba, „Przegląd Polityczny”, Nr. 99/2010, S. 115.
} 
nach Frankreich, wo er in Paris und Rennes als Deutschlehrer arbeitete. Als nächstes lehrte er Geschichte in den USA. Die in der Emigration verbrachten Jahre betrachtete er als „politische Lehrjahre”, die sich mit der Zeit als nützlich erweisen sollten. Er gewann damals, wie er mehrfach wiederholte ,eine Distanz zur Welt, der er entstammte, und lernte die Skepsis als Quintessenz der bürgerlichen Tugenden zu begreifen"».

1958 kehrte er nach Deutschland zurück, wo er zunächst in Münster den Lehrstuhl für Geschichte und anschließend in Stuttgart den für Politologie übernahm. Später zog er sich aus dem akademischen Wissenschaftsbetrieb zurück und ließ sich in Kilchberg bei Zürich nieder (dem letzten Wohnort Thomas Manns), von wo aus er aktiv am gesellschaftlichen, politischen und kulturellen Leben Deutschlands teilnahm. Die damaligen Geschehnisse bewertete er aus einem konservativen Blickwinkel. In den Sechziger- und Siebzigerjahren beteiligte er sich als Politologe an den Diskussionen über die Innenpolitik der Bundesrepublik Deutschland und prägte damit den öffentlichen Diskurs jener Zeit ${ }^{9}$. Einer besonderen Kritik unterzog er die Schulreform, die Studentenrevolution und die Politik des Staates gegenüber dem Terrorismus - dies alles aus einer konservativen Sicht, auch wenn zu bemerken ist, dass die Konservativen ihn stets für zu liberal, die Linken hingegen für zu konservativ hielten. Seine Suche nach dem eigenen Weg und seine Lebensentscheidungen bezahlte er mit depressiven Zuständen, von denen sein Biograf Urs Bitterli berichtet ${ }^{10}$.

Seine Liebe zur Geschichte erwachte schon in der Kindheit bei der Lektüre historischer Romane. Sein Bruder Klaus erwähnt in seinen Schriften, wie sich beide in einem Schrank einschlossen, und Golo ungeheure Geschichten erzählte ${ }^{11}$. In der Textsammlung Erinnerungen und Gedanken. Eine Jugend in Deutschland bekennt er, dass ihm ,als er kaum lesen konnte, das Lesebuch für die Grundschule nicht mehr genügte. (...) [Das Lesen] war ein völlig neues und wunderbares Gefühl (...), schöner noch als das spätere erste Fahrrad"12.

Ein Bild der Epoche und ein Porträt der Familie Mann zeichnete Golo Mann in seiner Autobiografie Erinnerungen und Gedanken. Eine Jugend in Deutschland (1986), deren Titel an die Erinnerungen Bismarcks anspielt. Andere Zeitzeugnisse sind Zeiten und Figuren. Schriften aus vier Jahrzehnten (1979) und Wir alle sind, was wir gelesen: Aufsätze und Reden zur Literatur (1989).

Golo Mann ist berühmt für seine Biografien wichtiger Personen der deutschen Geschichte. Besondere Anerkennung weckte sein Opus magnum, die 1971 veröffentlichte Wallenstein-Biografie ${ }^{13}$, die 1979 sogar verfilmt wurde. Der Autor bekannte, das dieses Werk seine immense Bedeutung der literarischen Qualität verdanke, darüber hinaus handele es sich um einen ,allzu wahren Roman”. 1947 veröffentlichte er eine Biografie von Friedrich von Gentz.

\footnotetext{
${ }^{8}$ Ebenda, S. 121.

${ }^{9}$ Vgl. „Borussia. Kultura. Historia. Literatura”, Nr. 44-45/2008 und Nr. 32, 2004, die der ganzen Familie Mann gewidmet sind.

${ }^{10}$ U. Bitterli, Golo Mann - Instanz und Außenseiter. Eine Biographie, Berlin 2004.

${ }^{11} \mathrm{Vgl}$. K. Mann, Punkt zwrotny (Wendepunkt), übers. M. Wydmuch, Warszawa 1993.

${ }^{12}$ G. Mann, Erinnerungen und Gedanken. Eine Jugend in Deutschland, Frankfurt a. M. 1986, S. 67. Auf den weiteren Seiten seiner Erinnerungen empfiehlt Golo Mann, Kindern nur gute Literatur zukommen zu lassen.

${ }^{13}$ Allein im ersten Jahr wurden über 100000 Exemplare des Wallenstein verkauft, eines Werks, das bei sehr kleinem Druck knapp 1400 Buchseiten einnimmt. Vgl. Wallenstein. Sein Leben erzählt von Golo Mann, Frankfurt a. M. 1971.
} 
Golo Mann starb im April 1994. Erwähnt sei eine wichtige Initiative der 2010 in Wiesbaden gegründeten Golo-Mann-Gesellschaft, die seit 2013 den Golo-Mann-Preis für ein Werk aus dem Bereich der Geschichtsschreibung vergibt.

\section{Die Bezüge zwischen Geschichte und Literatur}

Geschichtsschreibung war für Golo Mann eine große Kunst. Um ihr Geheimnis zu ergründen, zeigte er in seinem Essay Geschichtsschreibung als Literatur, wie viel sie mit Literatur gemein hat.

Was verbindet - nach Ansicht des Autors - diese beiden Bereiche? Wenn der Historiker Inhalte vermittelt, erzählt er wie ein Romanschriftsteller. Ähnlich wie dieser, hegt er die Ambition gelesen zu werden. Es mag wie ein Vorwurf klingen, aber die Historiker folgen den Schriftstellern auch darin, dass sie fabulieren. Thukydides verwendete in seinem berühmten Werk dramatisierte Dialoge (etwa den berühmten Melierdialog), die er nicht hatte hören können, aber die - wie er fand - so lauten sollten, wenn er sie gehört hätte (sic!). Auch heute suchen Historiker nach Gedanken, Ansichten, Überzeugungen, die ihren Ausführungen über konkrete Personen und Ereignisse Sinn verleihen. Dies ist geradezu eine Notwendigkeit, da andernfalls der Untersuchungsgegenstand kaum kohärent darzustellen wäre.

In politologischer Hinsicht ist wichtig, dass sowohl in der Geschichtsschreibung als auch der Literatur nur selten eine ,,vollkommene Einheit”, wie Golo Mann es nennt, auftritt. In beiden Sphären geht es um die Suche nach dem, was „ewig menschlich”, überzeitlich, universal ist, sogar wenn man von konkreten Fakten ausgeht. Daher sei die politikwissenschaftliche Bemerkung angebracht, dass sich dank der Suche nach dem Allgemeinen bestimmte politische Prinzipien formulieren lassen, indem man sie aus konkreten Ereignissen ableitet. So etwa das Gesetz von Lord Palmerston, dass es keine ewigen Feinde und keine ewigen Freunde gibt. Oder die Beobachtung, dass die Demokratie eine Neigung hat, sich in eine Diktatur zu verwandeln. Diese Gedenken entspringen der Suche nach dem, was sich als Gesetz der Wirklichkeit, als allgemeines Prinzip bezeichnen lässt.

Es mag erstaunlich anmuten, wird aber nach kurzem Nachdenken offenkundig, dass beide, Historiker und Schriftsteller, „Künstler, Magier, Produzenten des Scheins”14 sind. Es geht um die Schaffung eines Anscheins von Ganzheit. Mit einer gewissen ludischen Note führt Golo Mann das Beispiel des Hans Castorp an, dessen sieben Jahre auf dem Zauberberg auf 700 Buchseiten Platz finden. Ein anderes Beispiel wäre die Beschreibung der Geschichte eines Volkes oder eines Jahrtausends in einem ähnlichen Umfang. Dieses gemeinsame Phänomen bezeichnet Mann als „Perspektivverkürzung”. Dem Historiker und dem Romancier gemein ist das Treffen einer endlichen Auswahl aus einem unendlichen Material: „Wer vermöchte vollständig jene Unendlichkeit der Unendlichkeit darstellen, welche sich in dem Begriff «Europa im 18. Jahrhundert» verbirgt?"15.

${ }^{14}$ G. Mann, Dziejopisarstwo jako literatura (Geschichtsschreibung als Literatur), in: Ders., Ludzie myśli, ludzie władzy, historia (Mernschen des Gedankens, Menschen der Macht, Geschichte), übers. E. Paczkowska-Łagowska, Kraków 1997, S. 10.

${ }^{15}$ Ebenda, S. 11. 
Geschichte und Literatur sind Künste, die sich durch ähnliche Fantasie und Energie auszeichnen. In beiden Fällen kann als Inspiration eine Information, ein Tatsache der Wirklichkeit dienen. Für den Literaten kann dies etwa die Geschichte von unter Tage gefangenen Bergleuten sein, die sind, für den Historiker eine in einem Archiv gefundene Urkunde. Deshalb gilt es in beiden Fällen, Psychologe, genauer Beobachter der Wirklichkeit und ihr Arrangeur zu sein.

Welche Unterschiede bestehen zwischen Historiker und Schriftsteller? Der Geschichtsschreiber analysiert oft, verzichtet auf den schönen Schein, nimmt kritische Unterscheidungen vor, wobei es auch vorkommt, dass Schriftsteller die Sprache der schönen Literatur zugunsten eines Essays verlassen.

Unterschiedlich ist die Einstellung zur Frage der Wahrheit. Für den Historiker geht es um ihre Wiedergabe, während für den Schriftsteller die Unwahrheit oft ein unverzichtbares Mittel ist.

Trotz der Übereinstimmung bestimmter Themen und Motive unterscheidet den Historiker vom Romanschriftsteller, dass der schöne Schein nur ein Nebeneffekt seiner Arbeit ist, für den Literaten dagegen der Hauptzweck.

Der Historiker strebt nach der Wirklichkeit, ganz anders als der Romancier. Der Historiker muss sein eigenes Ich in den Schatten stellen; in den Vordergrund des Werks gerückt, würde es auf Ablehnung stoßen. Anders verhält es sich beim Schriftsteller, der sich selbst todernst nimmt und aus dem Vollen seiner eigenen Erlebnisse, Emotionen, Eindrücke schöpft.

Es ist interessant, dass viele Historiker auch als Schriftsteller tätig waren, etwa Voltaire, Schiller (Golo Manns Lieblingshistoriker), Wells. Das ist durchaus möglich, wenngleich nicht allzu häufig geglückt.

Dieser Vergleich bezieht sich nicht auf - wie Mann es nennt - die „Beamten der Geschichtswissenschaft”, „Institutsmitarbeiter” oder „Archivisten”, die sich eines amtlichen Schreibstils befleißigen. Natürlich verrichten auch sie wichtige Arbeit, aber nicht um sie geht es dem Autor des Otto von Bismarck.

Golo Manns gesammelte Überlegungen machen bei reiflicher Überlegung deutlich, dass dieser scheinbar schwer aufrechtzuerhaltende Vergleich von Geschichte und Literatur durchaus überzeugt. Erstaunlich ist die Erkenntnis, wie viel diese beiden Bereiche verbindet, denn unterstreichen wir: Zwischen ihnen besteht mehr Verbindendes als Trennendes. Ist die historische Wirklichkeit voller Kriege, Revolutionen und Aufstände nicht eine große Erzählung, ein mächtiges Theater mit authentischen Figuren? Wer eignet sich nach Aristoteles zum Helden eines Dramas? Feldherren, Politiker, Könige, Kirchenfürsten! Der Geschichtsschreiber muss die Fähigkeit haben, als großer Erzähler aufzutreten. Deshalb stellen manche die Frage, ob Golo Mann Historiker oder Schriftsteller war ${ }^{16}$. Bei der Entgegennahme des Büchner-Preises stellte er sich selbst als „historischer Literat” vor. Früher konnte ein Historiker nicht zu Renommee kommen, wenn er nicht die Tugenden des Gelehrten und des Künstlers miteinander verknüpfte. Gegen Ende des 19. Jahrhunderts aber begann der klassische Stil des Geschichtsschreibers zu verschwinden.

\footnotetext{
${ }^{16} \mathrm{http}$ //www.thomasmann.de/thomasmann/leben/seine_kinder/golo/231192 (Zugriff 2018-03-01). So sieht etwa Marcel Reich-Ranicki Golo Mann in erster Linie als Schriftsteller, nicht als Historiker.
} 


\section{Geschichtsschreibung und Demokratie}

Golo Mann sieht die Ursachen für das Abgehen vom Stil der Geschichtsschreibung als einer Kunst, die der Literatur nahe steht, im Heraufziehen der Epoche der erfüllten Demokratie. Früher, im 19. Jahrhundert, „,ümmerten sich die Historiker um die Geschichte in der Zeit ihres Entstehens, manchmal wandten sie die Vergangenheit auf eingehende Arbeiten über die Zukunft an"17. Golo Mann beschreibt in seinem Essay Geschichtsschreibung als Literatur die Amerikaner, die den Europäern hinsichtlich der Vollkommenheit der Demokratie voraus waren, aber auch im ,neuen Stil der historischen Forschung und Darstellung" "18. Anfänglich, nach der Erlangung der Unabhängigkeit, wurde noch im klassischen, europäischen Stil geschrieben - so verfassten ihre Werke John Motley und Henry Adams. Im frühen 20. Jahrhundert dagegen entstand die Konzeption der sogenannten New History, deren Hauptziel das Abgehen von Figuren wie Königen und Heerführern, sogar von der Politik und staatlichem Handeln, hin zu den Massen, den breiten Schichten der Gesellschaft war. Im Zentrum der Betrachtung stand nun der einfache Mensch (common man), sein Leben, die Art, wie er sich kleidete, seine Mahlzeiten einnahm, die Freizeit gestaltete, seine religiösen Überzeugungen und Praktiken. Ein Beispiel für diese Art von Geschichtsschreibung ist Charles Beards The Rise of American Civilization, ein Werk voller Informationen aus den verschiedensten Bereichen, das kaum auf politische Krisen und Wahlkämpfe eingeht und nichts mit hoher Literatur zu tun hat. In seinem Buch Vom Geist Amerikas. Eine Einführung in amerikanischen Denken und Handeln im Zwanzigsten Jahrhundert bemerkt Golo Mann, dass ,,die großen Menschen der Vergangenheit auch heute noch groß und zeitgenössisch sind"1".

Der Autor der Biografie Wilhelm II. fasst auf sentenzhafte Weise den Unterschied zwischen der alten und der mit der Demokratie verbundenen neuen Weise der Geschichtsschreibung zusammen: „Der Stil eines Geschichtswerks schöpft aus zwei Quellen: dem Geist des Gegenstands und dem Geist der Epoche, welcher der Historiker angehört" ${ }^{20}$. Wenn es auf der politischen Bühne an bedeutenden Persönlichkeiten mangelt, fällt es schwer, das Handeln ,,gemeiner Politikautomaten” im epischen Stil zu beschreiben. Schon Johan Huizinga hat bemerkt, dass die Geschichtsschreibung ihre Form verloren habe, weil auch die Geschichte dies getan habe. Golo Mann diagnostiziert, dass die Geschichte in Richtung Soziologie driftet, in Richtung Berichterstattung und Analyse. Früher wurde über Wenige für Wenige geschrieben, heute über den Jedermenschen, Everyman, für alle. Der Massenmensch möchte nicht von bedeutenden Persönlichkeiten lesen, da diese ihm in jeder Hinsicht fern sind, sondern von solchen wie er selbst. Ortega y Gasset hat es so formuliert: „Der gemeine Mensch, dazu angeleitet, beschloss selbst die Welt zu regieren"

Golo Mann analysiert auch den ehemaligen Charakter des Krieges, in dem es durchaus Platz gab für Schönheit und Theatralität. Dies datiert er von den Zeiten Homers bis zur

${ }^{17}$ G. Mann, Geschichte und Geschichten, Frankfurt a.M. 1962, S. 67.

${ }^{18}$ G. Mann, Dziejopisarstwo... (Geschichtsschreibung...), S. 23.

${ }^{19}$ G. Mann, Vom Geist Amerikas. Eine Einführung in amerikanischen Denken und Handeln im zwanzigsten Jahrhundert, Stuttgart 1961, S. 17.

${ }^{20}$ Ebenda, Dziejopisarstwo... (Geschichtsschreibung...), S. 24.

${ }^{21}$ J. O. y Gasset, Bunt mas (Der Aufstand der Massen), übers. P. Niklewicz, Warszawa 1995, S. 97. 
Frühzeit der Demokratie ${ }^{22}$. Damals gehörten Kriegsszenen zum geläufigen Kanon der Kunst. Im frühen 20. Jahrhundert änderte sich das. Niemand würde General Eisenhower bei der Überschreitung des Rheins malen, er würde sich nur lächerlich machen.

Ähnlich verhält es sich mit den Revolutionen des 20. Jahrhunderts, in denen es an spektakulären Stürmen und flammenden Reden mangelt. Die heutigen Revolutionen finden in Laboratorien und Forschungsinstituten, statistischen Ämtern, Plenarsälen und den Besprechungsräumen der Konzerne statt. Deshalb fällt es schwer, dieses Quellenmaterial mit nur einer verfügbaren Methodologie zu ergründen. Der Historiker benötigt also auch die Werkzeuge des Soziologen, des Politikwissenschaftlers, des Sozialpsychologen und des Volkswirts.

Es ist deutlich zu erkennen, wie sich mit der Entwicklung der Demokratie der Zeitgeist geändert hat, weshalb auch die Art und Weise, diese Geschichte zu erzählen, den Grundlagen der Analyse entsprechen muss: Golo Mann selbst jedoch vermeidet die Präsentation der Geschichte von der Seite der Strukturen oder der Regelhaftigkeit der Entwicklung, er „stellt uns die Geschichte von der Seite tatsächlicher Menschen aus Blut und Knochen dar" 23 .

Der Autor des Wallenstein bezieht sich auch auf die bekannte Konzeption vom „Ende der Geschichte", die ein Ende des freien Spiels schöpferischer Figuren und Ideen sowie das Ende der Geschichte als Drama oder Epos annimmt. ${ }^{24}$ Er polemisiert gegen diese Vision, indem er behauptet, dass die Geschichte dann zu einem geschlossenen fremden Museum würde. Verwandelt in eine Art Sozialkunde, wäre sie überflüssig wie Metaphysik. Nicht haltbar ist die These vom Ende der Geschichte aufgrund der Möglichkeit der Macht des Glaubens, politischer Leidenschaften und weltanschaulicher Fanatismen, die bewirken, dass sich über den Menschen nichts endgültig feststellen lässt. Und schließlich ist es ja der einzelne Mensch, der die Geschichte hervorbringt. Die Zeiten der Demokratie sind teilweise mit einem Verzicht auf Krieg als Werkzeug verbunden, von dem bis dato häufig Gebrauch gemacht wurde, was Mann zufolge Stil und Sozialgefüge der Epoche prägte ${ }^{25}$.

Welche Aufgaben stehen heutzutage vor dem Historiker? Wenn er nicht zum Soziologen oder Analytiker werden und dem Leser vergnügen berieten möchte, muss er nach neuen Lösungen suchen. Er darf nicht den Vorbildern seiner Lehrer oder - wie Golo Mann es in einem weiteren Rahmen fasst - den Mustern der Geschichtsschreibung des 19. Jahrhunderts verhaftet bleiben.

Der deutsche Geschichtsschreiber bekannte einst, dass er die jüngste Geschichte hasse, da sie „nicht imstande ist, den Schrecken in Schönheit oder wenigstens Literatur zu verwandeln" ${ }^{26}$. Nicht zu vergessen ist, dass die Griechen die Geschichte unter die Obhut der Muse Klio gestellt und so ihren poetischen Rang hervorgehoben haben. Aristoteles hat die Verbindung zwischen Geschichte und Poesie folgendermaßen formuliert: „(...) nicht Erzählung des Geschehenen (ist) Aufgabe des Dichters, sondern Erzählung der Begebenheiten, wie sie geschehen sein könnten, und des Möglichen nach der Wahrscheinlichkeit

${ }^{22}$ G. Mann, Dziejopisarstwo... (Geschichtsschreibung...), S. 27.

${ }^{23}$ E. Paczkowska-Lagowska, Golo Mann i sztuka dziejopisarstwa (Golo Mann und die Kunst der Geschichtsschreibung), in: G. Mann, Ludzie myśli... (Menschen des Gedankens...), S. 321.

${ }^{24}$ G. Mann, Dziejopisarstwo... (Geschichtsschreibung...), S. 29.

${ }^{25}$ Ebenda, S. 31.

${ }^{26}$ J. Fest, Szczęście... (Glück...), S. 124. 
oder Notwendigkeit. (...) der Geschichtsschreiber und Dichter unterscheiden sich (...) dadurch, dass der eine erzählt, was geschehen ist, der andere, wie es hätte geschehen können, deswegen ist die Poesie auch philosophischer und ernsthafter als die Geschichte. Denn die Poesie stellt mehr das Allgemeine, die Geschichte das Einzelne dar"27. Golo Mann dagegen träumte von Historikern, die als Schriftsteller gelten dürfen, also solchen, die versuchen, aus dem Einzelnen das Universale hervorzubringen.

\section{Der prophetische und besondere Charakter der Geschichte}

Dier ersten Konklusionen betreffen Golo Manns oben präsentierten Reflexionen aus dem analysierten Essay Geschichtsschreibung als Literatur. Aus den Gedankengängen des deutschen Denkers ergeben sich mehrere sehr wichtige Schlussfolgerungen. Zum ersten muss der Historiker so schreiben, dass er den Leser mitnimmt, also: schön. Zum Zweiten muss er über Menschen und für Menschen in einer zugänglichen Sprache schreiben. Es ist kein Zufall, dass etwa ein Buch über den Warschauer Aufstand zum Bestseller werden kann. Es geht um die schwierige Kunst, eine große Menge Fakten auf interessante Weise darzustellen. Natürlich erweckt dies Vorwürfe, vor allem seitens der Spezialisten, die es vorziehen würden, wenn sich der Autor einer wissenschaftlichen und nicht einer literarischen Werkstatt bedienen würde. Daher fällt es dem Historiker keineswegs leicht, seinem Handwerk nachzugehen. Er ist hin- und hergerissen zwischen Schönheit, literarischer Verarbeitung der Problematik, die ihm einen größeren Leserkreis beschert, und der Anerkennung der Fachkollegen, für die Fakten wichtiger sind als die Sprachebene. Golo Mann schrieb seine Werke mit einer ungeheuren, an Flaubert gemahnenden Sorgfalt. Sein Stil ist einfach, aber diese Einfachheit ergibt sich aus enormer Gelehrtheit, nicht aus Banalität. Niemals gab er sich als Historiker zu erkennen, schrieb über seine Figuren mit Distanz, auch wenn er oft von ihnen fasziniert war. In seinen Analysen bewahrte er stets einen hohen Grad an Objektivität.

Die Geschichte ist eine Kunst, die sich auf Fakten stützt, auf Wissen, aber andererseits auch zur Wirklichkeit hinführen soll und deshalb authentisch sein muss. Das Ziel des Geschichtsschreibers ist die Vermittlung von Wahrheit über Menschen auf der Grundlage mannigfaltiger Quellen. Der Sohn von Thomas Mann beschrieb sich selbst als Historiker, „der in den Kategorien eines unverwirklichten Erzählers zu sehen ist, das ist gewissermaßen das Geheimnis seines Lebens"28.

Golo Mann ist nicht naiv, er weiß, dass sich das Gewesene nicht so rekonstruieren lässt wie sie war, dass ein vollkommen objektives Bild der Vergangenheit unmöglich ist. Dem Historiker fällt die schwierige Aufgabe zu, Fragmente des Geschehenen zu interpretieren, nach dem verborgenen Sinn zu suchen. Dies bedeutet wiederum nicht, dass er imstande ist, Antworten auf die Frage nach dem Sinn der Geschichte zu liefern. Deshalb spricht er allein vom Schein des Ganzen, den die Geschichtsschreiber zu vermitteln vermögen.

Was die Geschichtsphilosophie angeht, steht der Autor des Otto von Bismarck auf dem Standpunkt, dass der Baustoff der Geschichte der Gedanke ist, und das ist ,gleichbedeutend

\footnotetext{
${ }^{27}$ Arystoteles, Poetyka (Poetik), 9 (1451b), übers. H. Podbielski, in: Ders., Dzieła wszystkie (Gesammelte Werke), Warszawa 2001, Bd. 6, S. 587-588.

${ }^{28}$ Ebenda.
} 
mit dem Erkennen einer rationalen Regelhaftigkeit des historischen Prozesses. Diese hingegen lässt sich erst post factum feststellen, da die Wirklichkeit voller Überraschungen steckt, die eine einfache Deutung des Geschichtsprozesses erschweren" 29 . Dennoch lässt sich sagen, das Golo Mann indirekt durchaus eine Art Philosophie der Geschichte präsentiert. Er behauptet, dass nicht alles in der Geschichte vernünftig ist, da in ihrem Zentrum der tatsächliche Mensch steht, der einzigartig, spontan, irrational ist. Von dieser „,menschlichen" Seite her beschreibt Golo Mann die Geschichte.

Zum Abschluss dieser Betrachtungen lohnt es sich, noch einmal auf die Einzigartigkeit der Geschichte als Werkzeug in den Politikwissenschaften einzugehen. Die ehrgeizige (und oft schier unmögliche) Aufgabe, die Vorgänge in der Welt der Politik vorherzusehen, lässt sich nicht angehen, wenn man nicht gut die Geschichte kennt. Wie andere bedeutende Historiker lehren, ist eine vertiefte Kenntnis der Vergangenheit notwendig, um sich zutrauen zu können, ein Szenario für die Entwicklung einer politischen Situation, sei es auf nationaler oder internationaler Ebene, zu schreiben. Durch das Finden analoger Situationen in der Vergangenheit lassen sich Schlussfolgerungen über die Auswirkungen politischer Handlungen in der Gegenwart ziehen ${ }^{30}$.

In diesem Sinne besitzt die Geschichte einen prophetischen Charakter. Golo Mann trägt der Tatsache Rechnung, dass die Geschichte in höherem Maße von der menschlichen Natur beherrscht wird als von der Dialektik des Fortschritts. Es ist die menschliche Natur, die der Geschichte immer wieder gebietet sich zu wiederholen. Dadurch können wir aber auch politische Theorien konstruieren, Prinzipien ableiten oder in einem gewissen Grade antizipieren - denn den Menschen erkennen wir durch die Geschichte.

Golo Mann beschreibt auch eine Gefahr für den Historiker. Was sind für ihn schlechte Geschichtsbücher? Solche, „die zwar schön geschrieben sind, aber ihren Gegenstand nicht adäquat umfassen, ihn nicht an die richtige Stelle rücken, oder solche, die diesen Anforderungen zwar gerecht werden, aber bei der Kunst der Darstellung versagen, oder solche, die weder dem einen noch dem anderen gerecht werden"31.

\section{Schlussbemerkungen}

Haben wir es heute mit einer solchen Art der Geschichtsschreibung zu tun, von der Golo Mann gesprochen hat? Wohl eher nicht. Eine solche Geschichtsschreibung bedarf einer enormen Bildung, die Bereitschaft und Fähigkeit über den Tellerrand des Fachgebiets zu schauen, und eine außergewöhnliches literarisches Talent. Wer wäre heut imstande, solche Untersuchungsgegenstände wie Geschichte, Politik, Philosophie, Literatur, Musik oder Malerei miteinander zu verknüpfen? Der Autor das Wallenstein vermochte dies. Was nicht bedeutet, dass man nicht versuchen sollte, dem von Golo Mann gegebenen Vorbild des Geschichtsschreibers nachzueifern. Er selbst war überzeugt, dass man nach dem Unmöglich streben soll, um das kleinste begehrte zu erreichen. Einser seiner Meister, nämlich

\footnotetext{
${ }^{29}$ E. Paczkowska-Łagowska, Golo Mann ... (Golo...), in: G. Mann, Ludzie... (Menschen ...), S. 345.

${ }^{30}$ In politischer Hinsicht ist es wichtig, die Beschränktheit der historischen Analogie zu berücksichtigen, denn jede Situation ist anders. Andererseits gilt es an ihre große Überzeugungskraft zu denken.

${ }^{31}$ G. Mann, Dziejopisarstwo... (Geschichtsschreibung...), S. 16-17.
} 
Max Weber, hat einmal gesagt: Vollkommen richtig ist der Satz, und alle historischen Erfahrungen belegen dies, dass man nicht das erreichen würde, was möglich ist, wenn nicht immer wieder in der Welt das Unmögliche gewagt worden wäre"32.

Es fällt umso schwerer, Golo Mann gerecht zu werden, als es nicht leicht ist, in seinem eigenen Fachgebiet einer Kenner zu sein. Die gewaltige Menge der jeweiligen Fachliteratur wirkt einschüchternd. Schreiben wir beispielsweise ein Suchwort in eine Internetsuchmaschine, und wir bekommen Abertausende (wenn nicht Millionen) von Treffern. Wie soll man das alles ergründen? Es kann demnach kaum von Belesenheit in verschiedenen Wissensgebieten die Rede sein, wenn wir natürlich von der authentischen Vertiefung einer Problematik und nicht einfach von oberflächlichen Kenntnissen sprechen. Umso beeindruckender ist die Breite der Horizonte des Autors des Friedrich von Gentz.

Bei all dieser Bildung, dem Stilgefühl und der Sentenzhaftigkeit von Golo Manns Schriften muss man sich allerdings bewusst bleiben, dass - wie Hegel lehrt - die Eule der Minerva in der Dämmerung auftaucht. Wenn es also möglich ist, sich einer derartigen Geschichtsschreibung zu befleißigen, dann nur im reifen Alter. Die Jugend lässt eine derartige Ansammlung von Wissen und das Durchdenken so vieler Aspekte der Wirklichkeit schlichtweg nicht zu.

Bemerkenswert ist, dass eben gerade in Deutschland der Geschichte eine Schlüsselbedeutung zugemessen wurde. Das bedeutendste Beispiel dafür ist natürlich das Werk Hegels. Dieser deutsche Philosoph sieht in der Geschichte das Wirken des Weltgeists, deshalb besitzt sie den höchsten Wert. Bogdan Suchodolski hat in seiner Textsammlung zur deutschen Seele geschrieben: Der Historismus ist eine der kennzeichnendsten Merkmale des deutschen Philosophie. In keinem anderen Land haben Überlegungen über Geschichte und die Historizität des menschlichen Lebens eine so große Rolle für die Philosophie gespielt" ${ }^{33}$.

\section{Bibliographie}

Arystoteles, Poetyka, 9 (1451b), przeł. H. Podbielski, [w:] idem, Dzieła wszystkie, t. 6, Warszawa 2001.

Fest J., Szczęście jako zasługa, przeł. A. i A. Klubowie, „Przegląd Polityczny” 2010, nr 99.

Gasset y J. O., Bunt mas, przeł. P. Niklewicz, Warszawa 1995.

Mann G., Dziejopisarstwo jako literatura, [w:] idem, Ludzie myśli, ludzie władzy, historia, przeł. E. Paczkowska-Łagowska, Kraków 1997.

Mann G., Erinnerungen und Gedanken. Eine Jugend in Deutschland, Frankfurt a. M. 1986.

Mann G., Geschichte und Geschichten, Frankfurt a. M. 1962.

Mann G., Vom Geist Amerikas. Eine Einführung in amerikanischen Denken und Handeln im zwanzigsten Jahrhundert, Stuttgart 1961.

Mann G., Wallenstein. Sein Leben erzählt von Golo Mann, Frankfurt a. M. 1971.

Mann G., Gaus G., Nienawidzę wszelkich skrajności, przeł. K. Zajas, „Przegląd Polityczny” 2010, nr 99.

${ }^{32}$ M. Weber, Polityka jako zawód i powołanie (Politik als Beruf und Berufung), übers. A. Kopacki, Kraków 1998, S. 110.

${ }^{33}$ B. Suchodolski, Dusza niemiecka w świetle filozofii (Die deutsche Seele im Lichte der Philosophie), Poznań 1947, S. 36. 
Mann K., Punkt zwrotny, przeł. M. Wydmuch, Warszawa 1993.

Schlegel F., „Athenäum” 1798, Nr. 80.

Anna Citkowska-Kimla, dr hab., prof. UJ. Holds degrees in politology, philosophy and Polish literature from Jagiellonian University; appointed in The Institute of Political Science and International Relations. Guest Lecturer in the University of Vienna. She carries out research in (especially German) political thought history and modern political movements and doctrines. She is interested in the paradigm of romantism in politics. Author oft he following selected publications: Romantyzm polityczny w Niemczech; Między sercem a rozumem. Romantyzm i realizm w polskiej myśli politycznej; Paradigm of political romanticism; The history of polish environmental protection movements. 